\title{
Olanzapin: Pharmakologie, Pharmakokinetik und Therapeutisches Drug Monitoring
}

TDM Arbeitsgruppe
der AGNP*
Federführend:
M. L. Rao ${ }^{1}$
C. Hiemke
K. Grasmäder
P. Baumann

Olanzapine: Pharmacology, Pharmacokinetics and Therapeutic Drug Monitoring

\section{Zusammenfassung}

Olanzapin ist ein gut wirksames und verträgliches atypisches Antipsychotikum. Es wird in der Leber zu großen Teilen N-glukuronidiert. Oxidationsreaktionen werden über das Cytochrom P450 Isoenzym CYP1A2 und in geringem Ausmaß über CYP2D6 katalysiert. Die Olanzapinserumspiegel streuen interindividuell sehr stark, sind aber über den Bereich der therapeutisch üblichen Tagesdosen linear. Sie werden durch Induktion von CYP1A2 bei Rauchern oder durch Carbamazepinkomedikation gesenkt. Sie steigen an nach Hemmung von CYP1A2 durch Fluvoxamin, jedoch nicht durch andere selektive Serotoninwiederaufnahmehemmer. Eine Hemmung von CYP2D6 durch andere Substanzen wird in Kombination mit weiteren Faktoren, wie weiblichem Geschlecht oder hohem Alter, klinisch relevant. Obwohl eine Dosisanpassung bei leichter bis mäßiger Leber- oder Niereninsuffizienz nicht erforderlich ist, empfiehlt sich bei solchen Patienten die therapeutische Serumspiegelüberwachung, um die Sicherheit einer Olanzapintherapie zu unterstützen. Für das TDM oder toxikologische Untersuchungen von Olanzapin sind verschiedene hochdruckflüssigchromatographische Methoden mit Ultraviolett- oder massenspektrometrischer Detektion etabliert worden. Als optimale Wirkkonzentration im Serum oder Plasma werden $20-40 \mathrm{ng} / \mathrm{ml}$ angesehen. Als Schwelle für eine erhöhte Wahrscheinlichkeit des Auftretens unerwünschter Ereignisse wird eine Konzentration von $80 \mathrm{ng} / \mathrm{ml}$ betrachtet. In neuerer Zeit wurde über das Auftreten von Neutropenien und Agranulozytosen unter Olanzapin-Therapie berichtet. Es zeigten sich unter Olanzapin des Weiteren ein mäßiger Anstieg der Prolaktin-

\section{Abstract}

Olanzapine is an effective and safe antipsychotic drug. Its pharmacokinetic properties are comparable to those of classical antipsychotics. Oxidative processes are mediated by the cytochrome P450 isoenzyme CYP1A2 and to a minor degree by CYP2D6. Olanzapine's main route of metabolism is by glucuronidation. Therapeutic doses result in a wide variability of serum levels; dose and serum concentration are linearly correlated. Smoking and carbamazepine induce cytochrome P450 isoenzymes and thus decrease olanzapine serum levels. Inhibition of CYP1A2 by fluvoxamine yields increased concentrations; however, clinically relevant CYP2D6 inhibition was observed only in combination with additional disposition factors, such as female gender or old age. As a rule dose adjustment is not necessary but moderate renal or hepatic impairment calls for control of serum levels to provide maximal safety during olanzapine therapy. Therapeutic drug monitoring (TDM) and toxicology studies are carried out by HPLC methods using UV or MS detection. The optimal therapeutic range of olanzapine serum levels is 20 to $40 \mathrm{ng} / \mathrm{ml}$. Concentrations of $80 \mathrm{ng} / \mathrm{ml}$ are considered threshold for the occurrence of adverse events; however, toxicological studies showed that postmortem plasma levels are higher than antemortem levels. Lethality of high olanzapine was only observed in combination with other drugs. Moderate increases of prolactin levels were detected during administration of olanzapine. In relation to olanzapine therapy, several case reports of neutropenia and agranulocytosis appeared in the literature. Weight gain in olanzapine-treated patients does not correlate with serum levels. Ol-

Institutsangaben

${ }^{1}$ Klinik und Poliklinik für Psychiatrie und Psychotherapie der Rheinischen Friedrich-Wilhelms-Universität Bonn (Direktor: Univ.-Prof. Dr. W. Maier)

${ }^{2}$ Psychiatrische Klinik der Universität Mainz (Komm. Direktor: Univ.-Prof. Dr. L. G. Schmidt)

${ }^{3}$ Unité de Biochimie et Psychopharmacologie Clinique, Département Universitaire de Psychiatrie Adulte, Prilly-Lausanne, Schweiz (Direktor : Univ.-Prof. Dr. P. Guex)

Fußnote

* Workshop der TDM-Arbeitsgruppe der Arbeitsgruppe für Neuropsychopharmakologie und Pharmakopsychiatrie in Bad Homburg v. d. Höhe

Korrespondenzadresse

Prof. Dr. M. L. Rao · Klinik und Poliklinik für Psychiatrie · Sigmund-Freud-Str. 25 · 53105 Bonn E-mail: m.l.rao@uni-bonn.de 
spiegel und eine deutliche Zunahme des Gewichts. Insgesamt scheint es sinnvoll, die Therapie von Patienten, die mit Olanzapin behandelt werden, mit TDM zu unterstützen, da die Effizienz der Wirkung gesteigert und die Sicherheit erhöht werden kann. anzapine response is augmented when patients' serum levels are titrated to 20 to $40 \mathrm{ng} / \mathrm{ml}$ thereby minimizing the occurrence of side effects, thus TDM is recommended for patients treated with olanzapine.

\section{Einleitung}

Olanzapin ist ein gut wirksames und verträgliches Antipsychotikum, das 1996 in der Europäischen Union zur Behandlung schizophrener Störungen eingeführt wurde. In einer Dosis von $5-20 \mathrm{mg}$ pro Tag ist es antipsychotisch wirksam und Plazebo überlegen [1-3]. Im Vergleich zu Haloperidol und anderen hochpotenten Neuroleptika ist Olanzapin in seiner Wirkung auf Negativsymptome günstiger und das Risiko des Auftretens extrapyramidalmotorischer Nebenwirkungen, einschließlich Spätdyskinesien, geringer $[1,4]$. Daher wird Olanzapin zur Gruppe der atypischen Antipsychotika gerechnet. In der vorliegenden Übersicht werden die Pharmakologie und Pharmakokinetik von Olanzapin dargestellt und wird der Frage nachgegangen, inwieweit die Effizienz und die Sicherheit einer Therapie mit dem atypischen Antipsychotikum durch Therapeutisches Drug Monitoring (TDM) verbessert werden kann.

\section{Pharmakologie}

Indirekte pharmakologische Ergebnisse zeigen, dass bei der Schizophrenie unterschiedliche neuronale Systeme beteiligt sind; vor allem handelt es sich um eine Dysfunktion dopaminerger und möglicherweise auch serotonerger Systeme im Zentralnervensystem. Alle Antipsychotika blockieren postsynaptische Dopamin- $\mathrm{D}_{2}$-Rezeptoren. Die therapeutisch erwünschten antipsychotischen Effekte kommen wahrscheinlich durch Wirkung auf mesolimbische und mesokortikale dopaminerge Systeme zustande, extrapyramidale Nebenwirkungen eher durch Hemmung nigrostriataler Systeme. Bei atypischen Antipsychotika wird postuliert, dass sie bevorzugt im mesolimbischen und mesokortikalen Bereich wirken und darüber hinaus postsynaptische Seroto$\operatorname{nin}_{2 \mathrm{~A}}$-Rezeptoren blockieren. Vermutlich ist letztere Eigenschaft für die Wirkung atypischer Antipsychotika auf die Negativsymptomatik der Schizophrenie von Bedeutung [5 - 7]. Viele atypische Neuroleptika binden darüber hinaus weniger stark an Dopamin$\mathrm{D}_{2}$-Rezeptoren als typische Antipsychotika (Tab.1). Entsprechend werden Clozapin und Quetiapin schnell, Olanzapin und Zi- prasidon weniger schnell und das klassische Neuroleptikum Haloperidol nur sehr langsam durch Dopamin vom Dopamin$\mathrm{D}_{2}$-Rezeptor verdrängt $[8,9]$.

Auch elektrophysiologisch wirkt Olanzapin unterschiedlich in verschiedenen dopaminergen Systemen [9]. Mesolimbische $\mathrm{A}_{10}$-Zellen zeigen eine dosisabhängige Zunahme der Spontanaktivität unter Olanzapin, nicht aber $\mathrm{A}_{9}$-Zellen (Kernbereich der nigrostriatalen dopaminergen Neurone). Dieser Befund ist konsistent mit dem geringen Risiko des Auftretens von Extrapyramidalsymptomen unter Olanzapin [10]. Darüber hinaus haben neuere Beobachtungen in vivo aufgezeigt, dass Olanzapin, anders als bei Untersuchungen in vitro, bevorzugt muskarinerge $\mathrm{M}_{2}$ - und nicht $\mathrm{M}_{1}$-Rezeptoren besetzt. Dies könnte die geringen anticholinergen Wirkungen von Olanzapin erklären und ein Hinweis für eine geringere Beeinflussung der kognitiven Leistungsfähigkeit sein [11]. PET-Studien sprechen dafür, dass unter üblichen Tagesdosen von 10-20 mg Dopamin- $\mathrm{D}_{2}$-Rezeptoren und Serotonin-Rezeptoren maximal besetzt sind [12]. Unter Einbeziehung atypischer Neuroleptika (Clozapin, Olanzapin) besteht eine lineare Beziehung zwischen der durchschnittlichen klinisch wirksamen Dosis und der Affinität von Neuroleptika zu Dopamin- $\mathrm{D}_{2}$-Rezeptoren [13].

\section{Analytik}

Für pharmakokinetische oder toxikologische Untersuchungen oder das TDM von Olanzapin stehen verschiedene chromatographische Methoden zur Verfügung [14-18]. Das derzeit schnellste Verfahren ist die Hochdruckflüssigchromatographie (HPLC) mit Säulenschaltung [17]. Dabei werden Serum oder Plasma nach Zentrifugation auf eine Vorreinigungssäule $(10 \times 2 \mathrm{~mm} \mathrm{I.D.})$ aufgetragen. Das Säulenmaterial (cyanopropylbeschichtetes Kieselgel) bindet Olanzapin. Proteine, Lipide und andere störende Matrixbestandteile werden ausgewaschen. Nach Wechsel des Eluenten wird das Arzneimittel von der Vorreinigungssäule auf die analytische Säule $(250 \times 4,6$ mm I.D., gefüllt mit C18 ODS Hypersil-Material, $5 \mu \mathrm{m}$ ) gespült und getrennt. Die Detektion er-

Tab. 1 Rezeptorbindungskonstanten Ki (mmol/l) neuerer Neuroleptika (nach Arnt, Skarsfeldt [9])

D1 D2

\begin{tabular}{|c|c|c|c|c|c|c|c|}
\hline & D1 & $D 2$ & $5-H T 2 A$ & M1 & Alpha1 & $H 1$ & $\operatorname{Dosis}(\mathrm{mg} / \mathrm{d})$ \\
\hline Olanzapin & 10 & 2,1 & 1,9 & 2,1 & 7,3 & 5,6 & $10-20$ \\
\hline Clozapin & 53 & 36 & 4,0 & 0,98 & 3,7 & 17 & $200-400$ \\
\hline Risperidon & 21 & 0,44 & 0,39 & $>5000$ & 0,69 & 88 & $4-8$ \\
\hline Haloperidol & 15 & 0,82 & 28 & 570 & 7,3 & $>730$ & $10-20$ \\
\hline Sertindol & 12 & 0,45 & 0,20 & 260 & 1,4 & 440 & $12-24$ \\
\hline Quetiapin & 390 & 69 & 82 & 56 & 4,5 & 21 & $50-750$ \\
\hline Ziprasidon & 9,5 & 2,8 & 0,25 & $>10000$ & 1,9 & 510 & $>60$ \\
\hline
\end{tabular}


folgt durch Messung der UV-Absorption bei $254 \mathrm{~nm}$. Das Verfahren erfasst Konzentrationen von $>5 \mathrm{ng} / \mathrm{ml}$ Olanzapin mit einer Präzision von 5-13\%.

Eine Methode mit sicherer Identifizierung von Olanzapin und hoher Sensitivität ist die Flüssigchromatographie mit massenspektrometrischer Detektion (LC-APCI-MS) [15]. Die Aufreinigung erfolgt durch Festphasenextraktion über eine aktivierte SPE-Kartusche (BondElut C18 $200 \mathrm{mg}$ ). Als interner Standard wird ein Ethylderivat des Olanzapins eingesetzt. Das konzentrierte Eluat wird in der mobilen Phase (Acetonitril-Ammoniumformiatpuffer, $50 \mathrm{mM}$, pH 3,0 [25:75]) aufgenommen und mittels HPLC über eine Superspher RP18-Säule getrennt. Die Detektion erfolgt massenspektrometrisch mit chemischer Ionisation unter Atmosphärendruck. Die untere Nachweisgrenze liegt bei $1 \mathrm{ng} / \mathrm{ml}$, nachweisbar sind Konzentrationen ab $0,5 \mathrm{ng} / \mathrm{ml}$, die Wiederfindungsrate beträgt $85 \%$. Das Signal-KonzentrationsVerhältnis ist im Bereich von 1-1000 ng/ml linear; die Präzision und Richtigkeit betragen 7 bzw. $99 \%$ bei $10 \mathrm{ng} / \mathrm{ml}$.

\section{Pharmakokinetik und Interaktionen}

Pharmakokinetisch ähnelt Olanzapin den klassischen Neuroleptika. Die Zeit bis zum Erreichen der maximalen Plasmakonzentration $\left(t_{\max }\right)$ von Olanzapin liegt bei $5,1 \pm 1,2 \mathrm{~h}$ (Mittelwert \pm Standardabweichung), die Halbwertszeit $\left(\mathrm{t}_{1 / 2}\right)$ ist mit $30 \mathrm{~h}(20-70 \mathrm{~h})$ (Mittelwert [Bereich]) eher lang. Olanzapin ist zu $93 \%$ an Plasmaeiweiße gebunden, die Bioverfügbarkeit wird durch einen hohen First-Pass-Effekt auf $57 \%$ geschätzt (keine i.v.-Daten verfügbar). Das Verteilungsvolumen liegt bei 10-201/kg [19]. Olanzapin wird auf verschiedenen Wegen metabolisiert (Abb.1), wobei das 10-N-Glukuronid, 4'-N-Desmethylolanzapin, Olanzapin-4'-Noxid sowie 2- und 7-Hydroxyolanzapin die wichtigsten Vertreter sind [20-22]. Der Hauptabbauweg von Olanzapin verläuft über die Glukuronidierung. Aufgrund der geringeren Konzentration im Vergleich zur Muttersubstanz wird den Metaboliten nur ein geringer Einfluss auf therapeutische Effekte oder Nebenwirkungen zugeschrieben. Plasmaspiegel von Olanzapin im Fließgleichgewicht betragen bei therapeutischen Dosen zwischen 5 und $100 \mathrm{ng} / \mathrm{ml}$ und stellen sich nach etwa einer Woche ein. Die Pharmakokinetik ist für therapeutische Dosen bis $20 \mathrm{mg} / \mathrm{Tag}$ linear.

Wie für andere Neuroleptika wird interindividuell eine große pharmakokinetische Variabilität gefunden, d.h. eine 26fache Streuung des Verhältnisses Serumkonzentration zu Dosis; der Mittelwert liegt bei $1,5 \mathrm{ng} / \mathrm{ml}$ pro $\mathrm{mg} / \mathrm{Tag}$. Für $80 \%$ der Patienten variiert das Verhältnis von Serumkonzentration zu Dosis in einem 5 fachen Bereich von 0,6 bis $3,1 \mathrm{ng} / \mathrm{ml}$ pro $\mathrm{mg} / \mathrm{Tag}[14,23]$. Bisherige Untersuchungen lassen bereits Voraussagen über die Serumspiegel zu. Frauen zeigen höhere Serumkonzentrationen als Männer und ältere Patienten höhere als jüngere. Raucher haben geringere Serumkonzentrationen als Nichtraucher; dies wird wie bei Clozapin auf Enzyminduktion des Cytochrom P450 Isoenzyms 1A2 (CYP1A2) zurückgeführt [24]. Man könnte jedoch auch über eine Induktion von Glucuronyltransferasen spekulieren, da diese den Hauptabbauweg von Olanzapin katalysieren. Auch durch eigene Untersuchungen an 223 Patienten, unter diesen 107 monotherapierte, konnte ein Effekt des Rauchens auf Olanzapinserumkonzentrationen gefunden werden (Gaertner et al., unveröffentlichte Ergebnisse). Die dosiskorrigierten Serum-

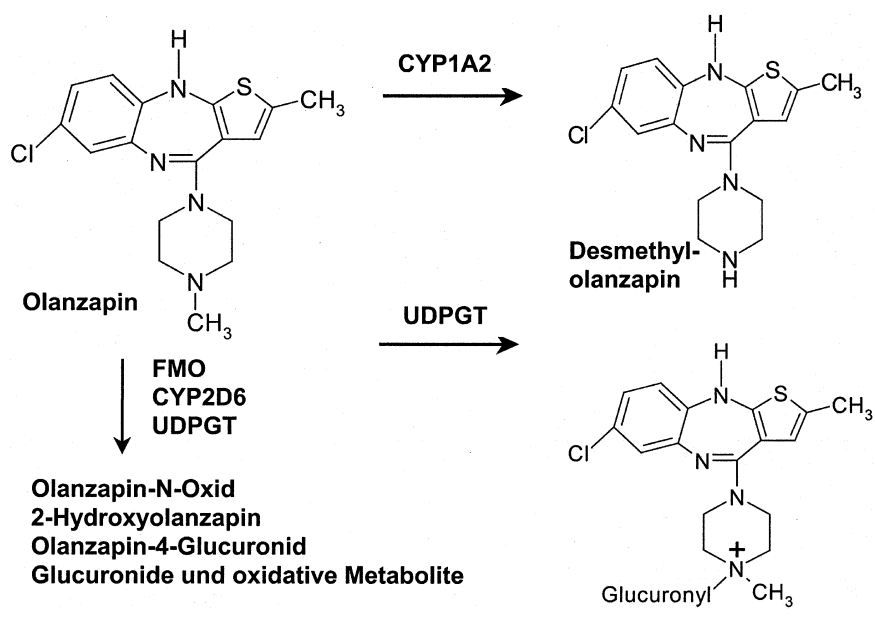

Abb. 1 Struktur und Metabolismus von Olanzapin und am Metabolismus beteiligte Enzyme: die Cytochrom P450 (CYP) Enzyme CYP1A2 und CYP2D6, Flavinmonooxygenase (FMO) und UDP-Glucuronyltransferase (UDPGT).

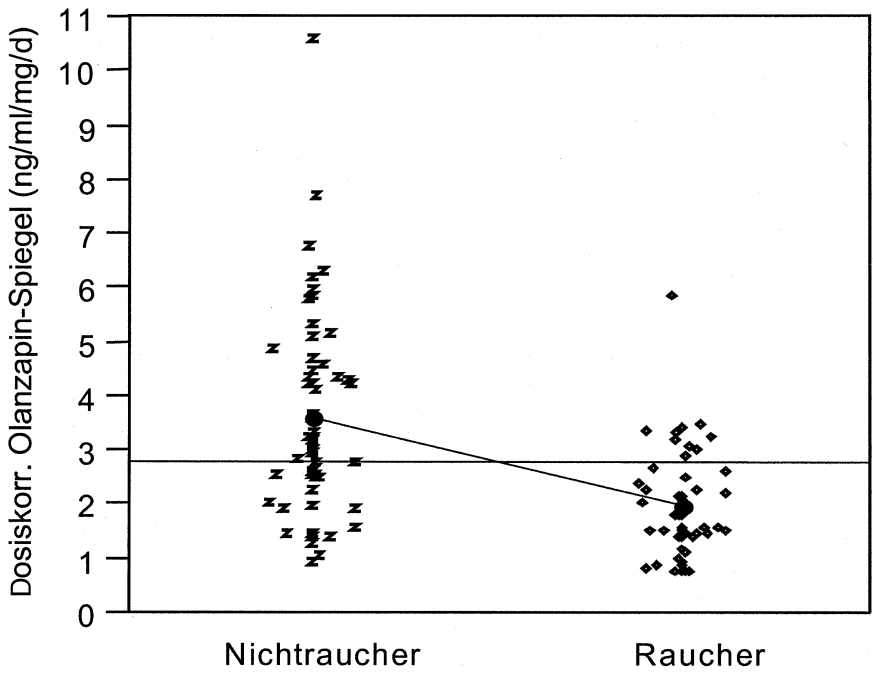

Abb. 2 Dosiskorrigierte Olanzapinspiegel bei Rauchern $(n=53)$ und Nichtrauchern $(n=56)$ unter Monotherapie. Die Variablen Alter und Geschlecht wurden ebenfalls berücksichtigt. Die Erniedrigung des Olanzapinspiegels durch Rauchen ist statistisch hochsignifikant $(p<0,0001)$ (Gaertner, unveröffentlicht).

spiegel lagen bei Rauchern konstant $50 \%$ niedriger als bei Nichtrauchern (Abb. 2). Wie bei anderen Neuroleptika senkt Carbamazepin die Serumspiegel von Olanzapin. Lucas u. Mitarb. führen das auf eine Induktion von CYP1A2 zurück [25]. Interessant wäre aber auch zu prüfen, inwieweit ein Nebenabbauweg über CYP3A4 infolge der bekannten Induktion durch Carbamazepin relevant wird. Komedikation mit trizyklischen Antidepressiva, Phenothiazin- und Thioxanthenneuroleptika steigert sie durch Inhibition des Cytochrom P450 Isoenzyms 2D6 (CYP2D6) $[20,21,23,24]$. Es existieren kaum Studien, bei denen Olanzapinspiegel vor und nach Zugabe einer Komedikation beim gleichen Patienten gemessen wurden, da in den meisten Berichten nur Gruppen von Patienten mit und ohne Komedikation untersucht wurden. Eine Ausnahme bildet ein interessanter Befund, wonach bei Absetzen der Carbamazepinkomedikation ein rascher und massiver Anstieg der Olanzapinspiegel beobachtet wird [26]. Von Interesse wären auch Untersuchungen zum Einfluss von 
Valproat, sowie Studien, in denen das Verhalten der Metabolite unter dem Einfluss interferierender Komedikationen mitbestimmt wird. Man könnte so Auskunft über deren Einfluss auf die einzelnen Abbauwege des Antipsychotikums bekommen. Tatsächlich fehlen klinische Interaktionsstudien bei Patienten unter Steady-State-Bedingungen, in denen Olanzapin und Metabolite bestimmt werden.

Ein Beleg dafür, dass CYP1A2 eher für den Metabolismus von Olanzapin relevant ist als CYP2D6, ergab sich aus Messungen von Olanzapinserumspiegeln, erhoben im Rahmen von TDM-Untersuchungen. Die Daten wurden retrospektiv ausgewertet zur Darstellung der Dosis-Serumspiegel-Beziehungen und zur Aufdeckung von Wechselwirkungen mit selektiven Serotoninwiederaufnahmehemmern. Die resultierenden Serumspiegel waren interindividuell hoch variabel (Abb.3). Komedikation zeigte folgende Einflüsse auf die Olanzapinspiegel: Wenn die Patienten als Begleitmedikament Fluvoxamin erhielten, von welchem inhibitorische Effekte auf CYP1A2 und andere CYP-Isoenzyme bekannt sind, waren bei durchschnittlich gleicher Tagesdosis die Olanzapinspiegel um 2,4fach höher als ohne Fluvoxamin. Die gleichzeitige Gabe von Paroxetin, einem potenter Inhibitor von CYP2D6, oder Sertralin zeigte keinen relevanten Einfluss auf die Olanzapinkonzentration (Abb. 3).

Untersuchungen an Patienten der Tübinger psychiatrischen Klinik zeigten bei Komedikation mit Sertralin oder Citalopram keinen Einfluss auf die Olanzapinserumspiegel, bei Kombination mit Paroxetin war bei einzelnen Individuen eine Tendenz zu erhöhten Serumkonzentrationen erkennbar (Gaertner et al., unveröffentlichte Ergebnisse). Kombinationen mit trizyklischen Neuroleptika (Perphenazin, Clopenthixol, Flupentixol, Fluphenazin) oder trizyklischen Antidepressiva (Amitriptylin, Trimipramin, Doxepin) sowie mit Butyrophenonen (Haloperidol, Benperidol) führen nach Tübinger Erfahrungen zu keinen relevanten Interaktionen. Im Gegensatz zu klassischen Antipsychotika wie Thioridazin oder Haloperidol hemmt Olanzapin, ähnlich wie die meisten neueren Antipsychotika, die Cytochrom P450 Isoenzyme in klinisch kaum relevanter Weise [27,28].

Da CYP2D6 nur eine geringe Rolle beim Metabolismus von Olanzapin spielt [29] ist davon auszugehen, dass die klinische Bedeutung dieser Einflüsse gering ist, wenn sie allein auftreten. Hinsichtlich der genetisch bedingten Variabilität der arzneistoffmetabolisierenden Cytochrom P450 Isoenzyme unterscheidet sich die Pharmakokinetik von Olanzapin bei Patienten, die eine genetische Defizienz von CYP2D6 aufweisen („poor metaboliser“), nicht von solchen, die ein intaktes Enzym besitzen („extensive metaboliser") [24]. Bei Kombination mehrerer sich addierender Faktoren sollte jedoch die Dosis, wie oben erwähnt, nach Kontrolle der Plasmaspiegel entsprechend angepasst werden.

Interethnische Unterschiede der Pharmakokinetik von Olanzapin wurden in einer Untersuchung an Chinesen, Kaukasiern und Japanern nicht nachgewiesen [24].

\section{Endolkrine Effekte}

Der Anstieg der Serumprolaktinkonzentrationen im Blut, vor allem zu Beginn einer Neuroleptikatherapie, lässt sich durch Blo-

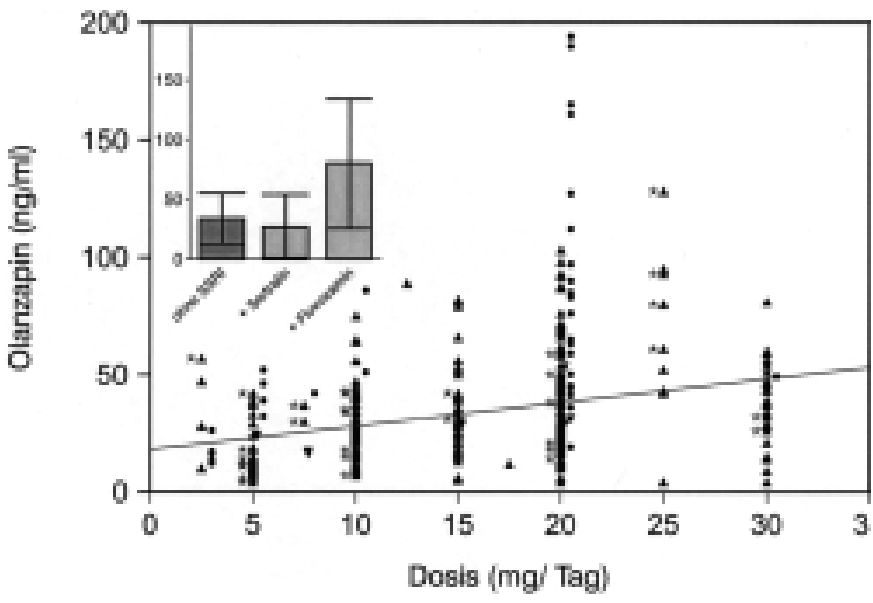

Abb. 3 Serumspiegel von Olanzapin in Abhängigkeit von der Tagesdosis ohne $(\boldsymbol{\Delta})$ und mit gleichzeitiger Gabe eines selektiven Serotoninrückaufnahmehemmers (SSRI), Fluvoxamin (๑), Sertralin (*) oder Paroxetin $(\boldsymbol{\nabla})$. Dargestellt sind Einzelwerte $(n=330)$ und Mittelwerte \pm SD der Patienten ohne SSRI oder andere Begleitmedikamente $(n=134)$ oder mit Fluvoxamin $(n=10)$ bzw. Sertralin $(n=21)$. Die mittleren Tagesdosen von Olanzapin waren ohne SSRI $16 \pm 7 \mathrm{mg}$, mit Sertralin $13 \pm 8 \mathrm{mg}$ und $18 \pm 7 \mathrm{mg}$ mit Fluvoxamin (Hiemke, unveröffentlicht).

ckade des Dopamin- $\mathrm{D}_{2}$-Rezeptors im tuberoinfundibulären System erklären, über den die negative Rückkopplung der Prolaktinfreisetzung durch Dopamin aus den laktotrophen Zellen der Adenohypophyse vermittelt wird. Dadurch kann es zu Galaktorrhö, Amenorrhö, Gewichtszunahme und sexuellen Funktionsstörungen kommen. Die antidopaminerge Aktivität von Neuroleptika bezüglich des Dopamin- $\mathrm{D}_{2}$-Rezeptors korreliert sowohl mit dem Serumspiegel der Neuroleptika als auch mit der Serumprolaktinkonzentration [13]. Auch PET-Rezeptorbindungsstudien mit Olanzapin im menschlichen Hirn zeigen eine dosis- und serumspiegelabhängige Bindung dieses Medikamentes an Dopamin- $\mathrm{D}_{2}$-Rezeptoren [30]. Innerhalb der atypischen neueren Neuroleptika ist der Einfluss auf den Prolaktinspiegel unterschiedlich stark ausgeprägt. Während der Einfluss von Clozapin marginal ist, kommen prolaktinbedingte Nebenwirkungen bei Benzamiden vergleichsweise häufig vor.

Um den Einfluss von Olanzapin auf die Prolaktinsekretion zu untersuchen, wurden die Serumprolaktinkonzentrationen von 205 schizophrenen Patienten aus Bonn und Essen unter einer Monotherapie mit Haloperidol, Clozapin, Olanzapin oder ohne Medikation verglichen (Rao et al., unveröffentlicht). Die Tagesdosen lagen für Haloperidol bei 2-25 mg (Mittelwert: $11 \mathrm{mg}$ ), für Clozapin bei $25-600 \mathrm{mg}$ (Mittelwert: $292 \mathrm{mg}$ ) und für Olanzapin bei 5-25 mg (Mittelwert: 14,3 mg). Damit korrelierte die mittlere Tagesdosis der drei Neuroleptika signifikant mit ihrer Affinität zum Dopamin- $\mathrm{D}_{2}$-Rezeptor, gemessen als Neuroleptische Einheit $(\alpha=0,05)$. Um Schwankungen aufgrund eines zirkadianen Rhythmus auszuschließen, wurden morgendliche Serumprolaktinkonzentrationen zur Auswertung herangezogen. Die Untersuchungsergebnisse von Frauen und Männern wurden in einer Varianzanalyse getrennt voneinander ausgewertet (Abb.4).

Die mittlere Serumprolaktinkonzentration der unbehandelten Frauen lag bei $0,279 \mathrm{nmol} / \mathrm{l}(\mathrm{n}=29)$, bei Behandlung mit Clozapin bei $0,478 \mathrm{nmol} / \mathrm{l}(\mathrm{n}=25)$, mit Haloperidol bei $1,863 \mathrm{nmol} / \mathrm{l}$ 

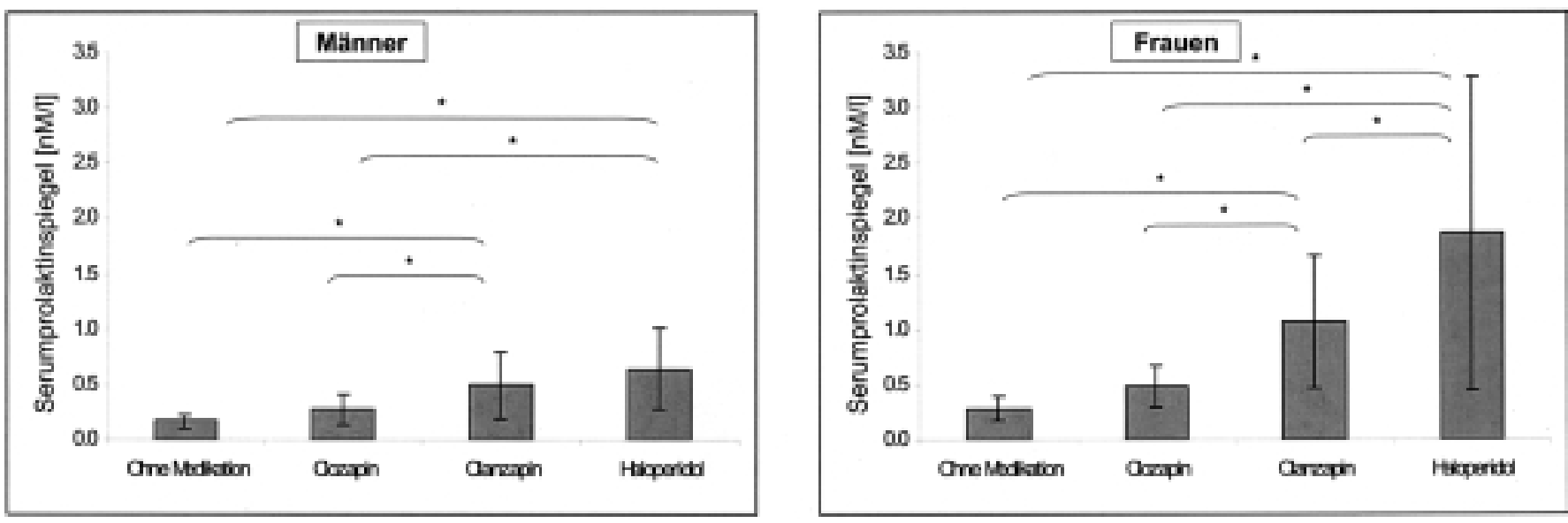

Abb. 4 Mittlere Serumprolaktinspiegel (+/-SD) in ng/ml bei unbehandelten Schizophrenen, unter Therapie mit Clozapin, Olanzapin oder Haloperidol, getrennt nach Männern und Frauen, $\alpha=0,05$ (Rao, unveröffentlicht).

$(\mathrm{n}=20)$ und mit Olanzapin bei $1,067 \mathrm{nmol} / \mathrm{l}(\mathrm{n}=27)$. Die Unterschiede der Prolaktinspiegel von Patientinnen unter Haloperidol, Olanzapin und Clozapin waren signifikant $(\alpha=0,05)$. Die Prolaktinspiegel unbehandelter Patientinnen unterschieden sich signifikant von denen unter Therapie mit Haloperidol und Olanzapin $(\alpha=0,05)$, jedoch nicht bei Behandlung mit Clozapin (Rao et al., unveröffentlichte Ergebnisse).

Bei männlichen Patienten wurden mittlere Serumprolaktinkonzentrationen von $0,167 \mathrm{nmol} / \mathrm{l}, 0,272 \mathrm{nmol} / \mathrm{l}, 0,638 \mathrm{nmol} / 1$ und $0,491 \mathrm{nmol} / \mathrm{l}$ ohne Therapie $(\mathrm{n}=26)$ bzw. unter Clozapin $(\mathrm{n}=29)$, Haloperidol $(n=25)$ und Olanzapin $(n=24)$ gemessen. Diese Unterschiede innerhalb der Gruppen waren signifikant $(\alpha=0,05)$, außer beim Vergleich Unbehandelter mit Clozapin sowie beim Vergleich Haloperidol versus Olanzapin bei therapierten Patienten. Der Prolaktinspiegelanstieg nach Olanzapin war deutlich, aber schwächer ausgeprägt als unter einer Haloperidolbehandlung. Zu dem gleichen Ergebnis kamen Crawford et al. [31], die darüber hinaus einen dosisabhängigen Anstieg der Serumprolaktinkonzentration zu Beginn der Olanzapintherapie finden, der sich jedoch im weiteren Therapieverlauf nivelliert.

\section{Einfluss auf das Körpergewicht}

Als bedeutende physiologische Veränderung wird bei Gabe von Olanzapin eine Zunahme des Körpergewichts bis zur 36. Woche nach Therapiebeginn beobachtet, die auf eine Blockade von Serotonin $_{2 c^{-}}$und Histamin ${ }_{1}$-Rezeptoren zurückgeführt wird [32]. Zusätzlich werden in letzter Zeit endokrine Faktoren, wie das Gewebshormon Leptin, der Tumornekrosefaktor $\alpha$ oder lösliche Tumornekrosefaktor-Rezeptoren mit der Gewichtszunahme unter Psychopharmakotherapie, insbesondere bei Olanzapin und Clozapin, in Zusammenhang gebracht [33-35].

In einer Metaanalyse zu Gewichtsveränderungen unter Neuroleptikagabe von Allison et al. [36] schätzt man die durch Olanzapin bedingte Gewichtszunahme auf im Mittel 4,2 kg innerhalb von 10 Wochen, die lediglich von Clozapin mit $4,5 \mathrm{~kg}$ in 10 Wochen übertroffen wird. Als Prädiktoren für die Gewichtszunahme unter Olanzapin wird ein niedriger Bodymass-Index und ein gutes Ansprechen auf die Olanzapintherapie identifiziert. Es kann jedoch keine Korrelation mit Olanzapinspiegeln im Serum gezeigt werden [31].

\section{Kardiale Effekte}

Eine besonders gefürchtete unerwünschte Arzneimittelwirkung, die auch mit atypischen Neuroleptika in Verbindung gebracht wird, ist deren Kardiotoxizität [37]. Sertindol wurde wegen solcher Eigenschaften vom Markt zurückgezogen. Die Analyse von EKG-Parametern unter Olanzapintherapie zeigt jedoch keinen signifikanten Unterschied der QTc-Intervall-Verteilung im Vergleich zu Plazebo [38].

\section{Toxische Effekte}

Zur Frage der potenziell toxischen Serumkonzentrationen von Olanzapin haben gaschromatographische Analysen von 22 forensischen Blutproben eine mittlere Olanzapinkonzentration von $318 \mathrm{ng} / \mathrm{ml}$ mit einer Spannbreite von $6 \mathrm{ng} / \mathrm{ml}$ bis $1600 \mathrm{ng} / \mathrm{ml}$ ergeben. Bei Untersuchung von 1655 Patientenproben aus der klinischen Routine lagen $70 \%$ der Werte unter $40 \mathrm{ng} / \mathrm{ml}$ mit einem Mittelwert von $36 \mathrm{ng} / \mathrm{ml}$ und einem Median von $26 \mathrm{ng} / \mathrm{ml}$, und nur 10\% der Konzentrationsangaben überstiegen $70 \mathrm{ng} / \mathrm{ml}$. Als potenziell toxisch werden von Robertson u. Mitarb. Konzentrationen $>80 \mathrm{ng} / \mathrm{ml}$ betrachtet [39].

Wong u. Mitarb. berichten von fünf Todesfällen unter Olanzapinmedikation [40]. Die Olanzapinkonzentrationen im Blut bewegten sich zwischen 190 und $1240 \mathrm{ng} / \mathrm{ml}$. Bei allen Fällen wurden noch weitere Medikamente nachgewiesen, d.h. bei keinem der Fälle wurde Olanzapin ursächlich mit dem Tod in Zusammenhang gebracht.

Sieben olanzapinassoziierte Todesfälle werden in der Studie von Levine u. Mitarb. beschrieben [41]; bei zwei Fällen führte man den Tod auf eine Olanzapinintoxikation zurück. Die Konzentrationen von Olanzapin im Blut lagen bei 160 und $980 \mathrm{ng} / \mathrm{ml}$. Die Wirkstoffkonzentrationen der fünf weiteren Todesfälle lagen im Bereich von 40 bis $270 \mathrm{ng} / \mathrm{ml}$. Diese Daten weisen darüber hinaus darauf hin, dass der Konzentrationsbereich postmortem höher 
liegt als für ante mortem gesammelte Blutproben im Fließgleichgewicht. Die Olanzapinkonzentration war unter Lagerung bei $4{ }^{\circ} \mathrm{C}$ nicht stabil.

In Bezug auf die Gewebsverteilung wird auch über 35 forensische Fälle berichtet [42]. Folgende Olanzapinkonzentrationen wurden gefunden: Herz $25-4800 \mathrm{ng} / \mathrm{ml}$, femorales Blut $25-1600 \mathrm{ng} / \mathrm{ml}$, Glaskörper $\quad 25-2100 \mathrm{ng} / \mathrm{ml}$, Leber $25-13000 \mathrm{ng} / \mathrm{ml}$, Galle $30-8400 \mathrm{ng} / \mathrm{ml}$, Urin $25-16000 \mathrm{ng} / \mathrm{ml}$. Olanzapin ist im Gewebe ebenfalls nicht stabil und sollte daher so schnell wie möglich analysiert werden. Bei zwei tödlichen Intoxikationen fanden Anderson u. Mitarb. folgende Gewebskonzentrationen: Blut (Herz) 4800 und $1300 \mathrm{ng} / \mathrm{ml}$, femorales Blut 1600 und $1200 \mathrm{ng} / \mathrm{ml}$, Leber 13000 und $8700 \mathrm{ng} / \mathrm{ml}$, Urin 16000 und $2200 \mathrm{ng} / \mathrm{ml}[42]$.

Zusammenfassend zeigt sich, dass die Datenlage hinsichtlich toxischer Serumkonzentrationen noch sehr heterogen ist. Da jedoch im Einzelfall bei Olanzapinserumspiegeln über $80 \mathrm{ng} / \mathrm{ml}$ mit toxischen Effekten zu rechnen ist, sollte diese Grenze möglichst nicht überschritten werden.

\section{Hämatotoxische Effekte}

Erste Erfahrungen zu Blutbildveränderungen unter Therapie mit Olanzapin führten im Februar diesen Jahres zu einer Meldung der Arzneimittelkommission [43]. Basierend auf sieben in der Literatur beschriebenen Fälle und der Auswertung von 384 Verdachtsmeldungen $\mathrm{zu}$ olanzapininduzierten Nebenwirkungen des Spontanerfassungssystems der Arzneimittelkommission der deutschen Ärzteschaft (AkdÄ) und des Bundesinstitutes für Arzneimittel und Medizinprodukte (BfArM), wurde seitens der AkdÄ die Empfehlung ausgegeben, „vor Behandlungsbeginn die Patienten über klinische Zeichen einer möglichen Blutbildveränderung zu informieren und das Blutbild der Patienten regelmäßig in zwei- bis vierwöchigen Abständen zu kontrollieren“ [43].

Unter den Fallberichten befanden sich einer über Agranulozytose, zwei über Neutropenien und einer über eine Leukopenie; die Verläufe waren nach Absetzen des Olanzapins reversibel, ein asymptomatischer Verlauf wurde jedoch nur in 2 Fällen beschrieben [44-47]. In einem Fallbericht remittierte eine olanzapinbedingte Granulozytopenie trotz fortbestehender Olanzapinmonotherapie [48].

\section{Therapeutisches Drug Monitoring}

TDM wird eingesetzt, um Unterdosierungen mit der Konsequenz mangelhaften Therapieansprechens oder Überdosierungen und daraus resultierende Intoxikationen zu vermeiden. Voraussetzung für den Einsatz von TDM ist jedoch die Kenntnis eines therapeutisch wirksamen Plasmaspiegels, dem Zielbereich, auf den man die Patienten einstellen sollte. Unter Olanzapin werden bei wirksamen Tagesdosen von 5-30 mg Plasmaspiegel zwischen 8 und $47 \mathrm{ng} / \mathrm{ml}$ festgestellt [23]. Ein therapeutischer Bereich von $9-45 \mathrm{ng} / \mathrm{ml}$ wurde für Olanzapin erstmalig von Perry u. Mitarb. 1997 ermittelt [49] und damit ein Nutzen von TDM zur Verbesserung der Therapie mit Olanzapin wahrscheinlich gemacht. Die Autoren berichten, dass oberhalb von Olanzapinplasmaspiegeln

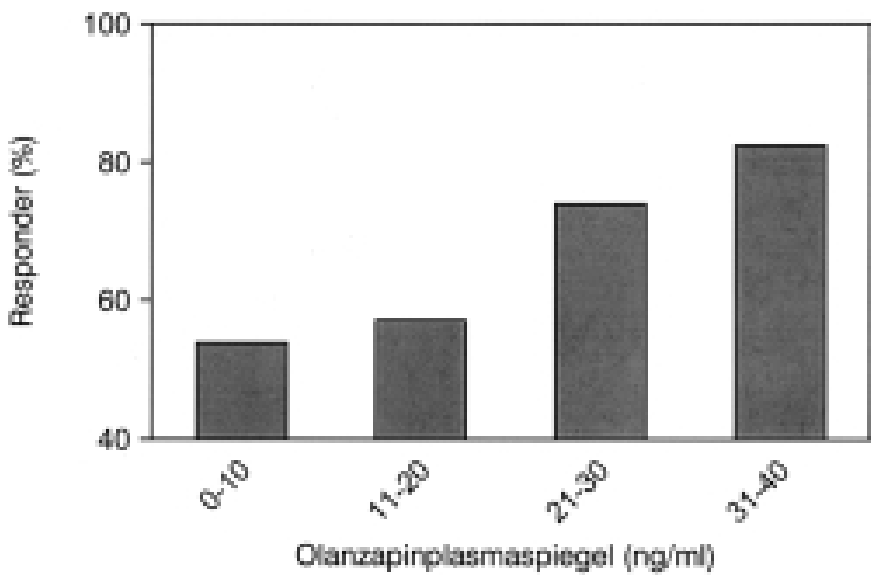

Abb. 5 Klinisches Ansprechen von Patienten, die mit Olanzapin behandelt wurden. Die Beurteilung erfolgte nach der Brief Psychiatric Rating Scale (BPRS). Als Responder wurden die Patienten definiert, die nach der BPRS-Skala mindestens um 30\% gebessert waren. Die Ergebnisse entstammen einer Nachauswertung von Daten der Literatur [24]. Die Patienten wurden nach Olanzapin-Plasmaspiegel-Bereichen in Gruppen sortiert und für jede Gruppe der Anteil an Respondern bzw. verschlechterten Patienten berechnet (Hiemke, unveröffentlicht).

von $9,3 \mathrm{ng} / \mathrm{ml}$ das Therapieansprechen besser ist als unterhalb dieser Grenze. Die Spiegel waren in der Regel 24 Stunden nach der letzten Einnahme von Olanzapin gemessen worden. Eine neuere Untersuchung an 84 Patienten, bei denen Olanzapinspiegel 12 Stunden nach der letzten Dosis im Plasma gemessen wurden, berichtet Schwellenwertspiegel von $23 \mathrm{ng} / \mathrm{ml}$ [50]. Ein ähnlicher Bereich lässt sich durch Nachauswertung von Ergebnissen von Callaghan et al. ermitteln [24]. Nach Regressionsanalyse der Brief Psychiatric Rating Scale (BPRS)-Scores gegen Olanzapinplasmaspiegel von über 300 Patienten, die mit fixen Dosen von Olanzapin behandelt wurden, schlussfolgerten die Untersucher keinen Zusammenhang zwischen Plasmaspiegeln und klinischem Ansprechen. Berechnet man jedoch den prozentualen Anteil der Responder in Abhängigkeit vom Plasmaspiegelbereich, dann erkennt man einen Zusammenhang (Abb. 5). Nach dieser Auswertung beträgt die Wahrscheinlichkeit des therapeutischen Ansprechens auf Olanzapin durch Einstellung der Plasmaspiegel auf $>21 \mathrm{ng} / \mathrm{ml} 78$ gegenüber $54 \%$ bei niedrigeren Spiegeln. Ein solcher Schwellenwert wird auch durch die PET-Studie von Kapur et al. bestätigt [12].

Neben forensischen Überlegungen eines toxikologischen Schwellenwertes bei $80 \mathrm{ng} / \mathrm{ml}$ lässt sich ein optimaler therapeutischer Bereich auch aus eigenen retrospektiven Analysen für Olanzapinserumspiegel zwischen $20-40 \mathrm{ng} / \mathrm{ml}$ nachvollziehen (Abb. 6). Messungen von Olanzapinserumspiegeln an insgesamt 165 Patienten (Hiemke et al., unveröffentlicht), erhoben im Rahmen des TDM, wurden retrospektiv ausgewertet. Die Tagesdosen betrugen zwischen 2,5 und $30 \mathrm{mg}$. Am häufigsten (37\% der Patienten) wurden $20 \mathrm{mg}$ Olanzapin verordnet. Die klinische Besserung, eingeschätzt nach Clinical Global Impression (CGI), war bei über $80 \%$ der Patienten mit Serumspiegeln zwischen 20 und $40 \mathrm{ng} / \mathrm{ml} \mathrm{zu}$ beobachten (Abb.6). Extrapyramidal-motorische Störungen wurden sehr selten berichtet, bei Olanzapinserumspiegeln über $40 \mathrm{ng} / \mathrm{ml}$ waren sie allerdings etwa doppelt so häufig wie bei niedrigeren Spiegeln. Aufgrund der an der Psychiatrischen Klinik der Universität Mainz gemachten Erfahrungen werden somit die von Perry u. Mitarb. [49,50] publizierten Ergebnis- 

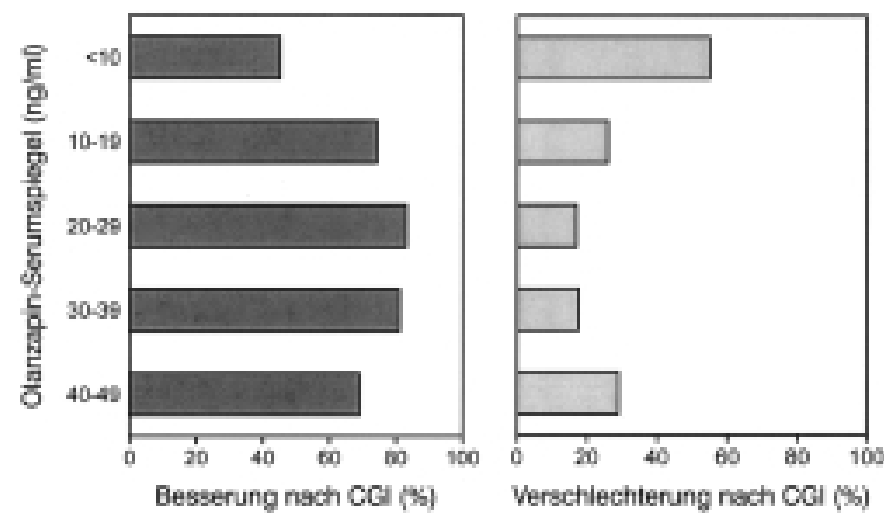

Abb. 6 Klinische Besserung und Verschlechterung von Patienten $(n=106)$, die mit Olanzapin behandelt wurden. Die Beurteilung erfolgte nach Clinical Global Impression (CGI). Zur Darstellung der Besserung wurden die Responder berechnet und als Responder die Patienten definiert, die nach der CGI-Skala wenig, viel oder sehr viel besser angegeben wurden. Als verschlechtert wurden solche Patienten definiert, die unverändert, etwas, viel oder sehr viel schlechter beurteilt wurden. Die Patienten wurden nach Olanzapin-Serumspiegel-Bereichen in Gruppen sortiert und für jede Gruppe der Anteil an Respondern bzw. verschlechterten Patienten berechnet (Hiemke, unveröffentlicht).

se mit einer schlechteren Response bei Olanzapinspiegeln unterhalb von $20 \mathrm{ng} / \mathrm{ml}$ und noch ausgeprägter unterhalb von $10 \mathrm{ng} /$ $\mathrm{ml}$ bestätigt.

Eine Dosisanpassung unter TDM-Kontrolle ist bei Beeinträchtigung der renalen Elimination nicht notwendig, da der Anteil von renal ausgeschiedenem Olanzapin nur gering ist. Olanzapinspiegel werden auch nicht durch Dialyse beeinflusst. Die Dosis muss bei leichter Leberinsuffizienz ebenfalls nicht angepasst werden. Es wurde sogar gezeigt, dass auch eine stärkere Beeinträchtigung der Leberfunktion, wie etwa bei zirrhotischen Patienten, die Plasmaspiegel von Olanzapin nicht verändert. TDM ist aber bei diesen Patienten trotzdem zu empfehlen, da sie häufig Begleitmedikation erhalten, die mit der Pharmakokinetik von Olanzapin interferieren kann.

Aus dem Interaktionspotenzial von Olanzapin ist zu folgern, dass TDM von Olanzapin bei solchen Patienten indiziert ist, die Begleitmedikamente erhalten, die CYP1A2 hemmen. Dies ist klinisch relevant für Fluvoxamin, welches ein potenter Inhibitor von CYP1A2 ist. Die Olanzapinspiegel werden im Mittel durch Fluvoxamin verdoppelt.

Zum Einfluss von Carbamazepin wurden in Tübingen 107 Patienten mit Olanzapinmonotherapie und 22 Patienten mit Olanzapin und Carbamazepin ohne weitere Komedikation untersucht (Gaertner et al., unveröffentlicht). Berücksichtigt man in einer linearen Regression des dosiskorrigierten Olanzapinspiegels (Zielvariable) neben den Faktoren Rauchen, Geschlecht und Alter den Einfluss von Carbamazepin, so lässt sich im geometrischen Mittel eine Senkung um 20\% durch die Komedikation nachweisen, dieser Unterschied ist zwar statistisch nicht signifikant $(p=0,08)$, der Trend wird aber durch andere Untersuchungen bestätigt [25].

Da es kaum möglich ist, den Einfluss der oben genannten Variablen auf den Serumspiegel von Olanzapin für den individuellen
Patienten vorab einzuschätzen, erscheint es sinnvoll, TDM bei Rauchern oder Patienten mit enzyminduzierenden oder -inhibierenden Medikamenten zu nutzen.

\section{Schlussbetrachtung}

Olanzapin ist ein atypisches Antipsychotikum, welches heute einen wichtigen Platz in der Therapie von Psychosen einnimmt. Es ist zu begrüßen, dass mehrere Untersuchungen den Nutzen von Plasmaspiegelbestimmungen von Olanzapin für die Therapieoptimierung belegen, wobei der therapeutische Bereich bei $20-40 \mathrm{ng} / \mathrm{ml}$ liegt. In der Literatur werden verschiedene Methoden (meistens HPLC) beschrieben, die die quantitative und zuverlässige Bestimmung von Olanzapin im Plasma erlauben. Olanzapin wird seit kurzem auch für die Behandlung und Prophylaxe der Manie empfohlen. Studien zum therapeutischen Bereich von Olanzapinplasmaspiegeln bei dieser Indikation sind deshalb von Interesse.

Interaktionsstudien belegen, dass vor allem CYP1A2-Hemmer die Plasmaspiegel von Olanzapin erhöhen und CYP1A2-Induktoren (Rauchen) diese erniedrigen. Bei zukünftigen Interaktionsstudien oder pharmakogenetischen Untersuchungen sollten auch die Plasmaspiegel der wichtigsten Metabolite unter SteadyState-Bedingungen bei Patienten gemessen werden, um die Rolle der einzelnen Cytochrom P450 Isoenzyme beim Metabolismus von Olanzapin besser zu verstehen. Abschließend wird festgestellt, dass aufgrund der bisherigen Datenlage Plasmaspiegelbestimmungen von Olanzapin zur Therapieoptimierung gerechtfertigt sind.

Gefördert durch das Bundesministerium für Bildung und Forschung.

\section{TDM-Arbeitsgruppe der AGNP}

Arbeitsgruppe für Neuropsychopharmakologie und Pharmakopsychiatrie; Vorsitzender: Prof. Dr. F. Holsboer;

Vorsitzender der Arbeitsgruppe TDM: Prof. Dr. P. Baumann

Mitglieder der Arbeitsgruppe: Prof. Dr. P. Baumann, Prilly-Lausanne/Schweiz, Dr. G. Eckermann, Kaufbeuren, Dr. I. Gaertner, Tübingen, Prof. Dr. C. Hiemke, Mainz, Dr. H.-J. Kuss, München, Prof. Dr. G. Laux, Wasserburg, Gabersee, Prof. Dr. B. Müller-Oerlinghausen, Berlin, Prof. Dr. M. L. Rao, Bonn, Prof. Dr. P. Riederer, Würzburg, Dr. S. Ulrich, Magdeburg, Prof. Dr. G. Zernig, Innsbruck. Weiterer Mitarbeiter: Prof. Dr. M. Bogusz, Riyadh

\section{Literatur}

${ }^{1}$ Beasley Jr CM, Tollefson G, Tran P, Satterlee W, Sanger T, Hamilton S and the Olanzapine HGAD Study Group. Olanzapine versus placebo and haloperidol. Acute phase results of the North American doubleblind olanzapine trial. Neuropsychopharmacology 1996; 14: 111-123

${ }^{2}$ Goldstein JM. The new generation of antipsychotic drugs: how atypical are they? Int J Neuropsychopharmacology 2000; 3: 339-349

${ }^{3}$ Martényi F, Metcalfe S, Schausberger B, Dossenbach MRK. An efficacy analysis of olanzapine treatment data in schizophrenia patients with catatonic signs and symptoms. J Clin Psychiatry 2001; 62 (Suppl. 2): $25-27$ 
${ }^{4}$ Tollefson GD, Sanger TM, Lu Y, Thieme ME. Depressive signs and symptoms in schizophrenia. A prospective blinded trial of olanzapine and haloperidol. Arch Gen Psychiatry 1998; 55: 250-258

${ }^{5}$ Richelson E. Receptor pharmacology of neuroleptics: relation to clinical effects. J Clin Psychiatry 1999; 60 (Suppl. 10): 5-14

${ }^{6}$ Meltzer HY. The role of serotonin in antipsychotic drug action. Neuropsychopharmacology 1999; 21: 106-115

${ }^{7}$ Kinon BJ, Lieberman JA. Mechanisms of action of atypical antipsychotic drugs: a critical analysis. Psychopharmacology 1996; 124: 2-34

8 Seeman P, Tallerico T. Rapid release of antipsychotic drugs from dopamine D2 receptors: an explanation for low receptor occupancy and early clinical relapse upon withdrawal of clozapine or quetiapine. Am J Psychiatry 1999; 156: 876-884

${ }^{9}$ Arnt J, Skarsfeldt T. Do novel antipsychotics have similar pharmacological characteristics? A review of the evidence. Neuropsychopharmacology 1998; 18: 63-101

10 Tollefson GD, Beasley jr CM, Tamura RN, Tran PV, Potvin JH. Blind, controlled, long-term study of the comparative incidence of treatmentemergent tardive dyskinesia with olanzapine or haloperidol. Am J Psychiatry 1997; 1254: $1248-1254$

${ }^{11}$ Raedler TJ, Knable MB, Jones DW, Lafargue T, Urbina RA, Egan MF, Pickar D, Weinberger DR. In vivo olanzapine occupancy of muscarinic acetylcholine receptors in patients with schizophrenia. Neuropsychopharmacology 2000; 23: 56-68

12 Kapur S, Zipursky RB, Remington G, Jones C, DaSilva J, Wilson AA, Houle S. 5-HT2 and D2 receptor occupancy of olanzapine in schizophrenia: a PET investigation. Am J Psychiatry 1998; 155: 921 - 928

13 Rao ML, Oades RD, Bagli M. Prolaktinantwort und psychophysiologische Reaktion auf Neuroleptika bei Frauen und Männern. In: RiecherRössler A, Rohde A. Psychische Erkrankungen bei Frauen. Freiburg: Karger, 2001: 168-180

${ }^{14}$ Aravagiri M, Ames D, Wirshing WD, Marder SR. Plasma level monitoring of olanzapine in patients with schizophrenia: Determination by HPLC with electrochemical detection. Ther Drug Monit 1997; 19: 307-313

15 Bogusz MJ, Kruger KD, Maier RD, Erkwoh R, Tuchtenhagen F. Monitoring of olanzapine in serum by liquid chromatography-atmospheric pressure chemical ionization mass spectrometry. J Chromatogr B Biomed Sci Appl 1999; 732: 257-269

${ }^{16}$ Catlow J, Barton R, Clemens M, Gillespie T, Goodwin M, Swanson S. Analysis of olanzapine in human plasma utilizing reversed-phase high-performance liquid chromatography with electrochemical detection. J Chromatogr B Biomed Sci Appl 1995; 668: 85-90

17 Weigmann H, Härtter S, Maehrlein S, Kiefer W, Krämer G, Dannhardt G, Hiemke C. Simultaneous determination of olanzapine, clozapine and demethylated metabolites in serum by on-line column switching high performance liquid chromatography. J Chromatogr B Biomed Sci Appl 2001; 759: 63-71

18 Raggi MA, Casamenti G, Mandrioli R, Volterra V. A sensitive high-performance liquid chromatographic method using electrochemical detection for the analysis of olanzapine and desmethylolanzapine in plasma of schizophrenic patients using a new solid-phase extraction procedure. J Chromatogr B Biomed Sci Appl 2001; 750: 137-146

19 Ereshefsky L. Pharmacokinetics and drug interactions: Update for new antipsychotics. J Clin Psychiatry 1996; 57 (Suppl. 11): 12-25

${ }^{20}$ Bever KA, Perry PJ. Olanzapine: A serotonin-dopamine-receptor antagonist for antipsychotic therapy. Am J Health Syst Pharm 1998; 55: $1003-1016$

${ }^{21}$ Broich K. Olanzapin. Ein neues Neuroleptikum mit „atypischem“ Wirkungsprofil. Arzneimitteltherapie 1997; 15: $33-37$

${ }^{22}$ Kassahun K, Mattiuz E, Nyhart jr E, Obermeyer B, Gillespie T, Murphy A, Goodwin RM, Tupper D, Callaghan JT, Lemberger L. Disposition and biotransformation of the antipsychotic agent olanzapine in humans. Drug Metab Dispos 1997; 25: 81 - 93

${ }^{23}$ Olesen OV, Linnet K. Olanzapine serum concentrations in psychiatric patients given standard doses: The influence of comedication. Ther Drug Monit 1999; 21: 87-90

${ }^{24}$ Callaghan JT, Bergstrom RF, Ptak LR, Beasley CM. Olanzapine: Pharmacokinetic and pharmacodynamic profile. Clin Pharmacokinet 1999; 37: $177-193$

${ }^{25}$ Lucas RA, Gilfillan DJ, Bergstrom RF. A pharmacokinetic interaction between carbamazepine and olanzapine: observations on possible mechanism. Eur J Clin Pharmacol 1998; 54: 639-643

${ }^{26}$ Licht RW, Olesen OV, Friis P, Laustsen T. Olanzapine serum concentrations lowered by concomitant treatment with carbamazepine. J Clin Psychopharmacol 2000; 20: 110-112
${ }^{27}$ Ring BJ, Binkley SN, van den Branden M, Wrighton SA. In vitro interaction of the antipsychotic agent olanzapine with human cytochromes P450 CYP2C9, CYP2C19, CYP2D6 AND CYP3A. Br J Clin Pharmacol 1996; 41: 181 - 186

28 Prior TI, Chue PS, Tibbo P, Baker GB. Drug metabolism and atypical antipsychotics. Eur Neuropsychopharmacol 1999; 9: 301 - 309

${ }^{29}$ Ring BJ, Catlow J, Lindsay TJ, Gillespie T, Roskos LK, Cerimele BJ, Swanson SP, Hamman MA, Wrighton SA. Identification of the human cytochromes P450 responsible for the in vitro formation of the major oxidative metabolites of the antipsychotic agent olanzapine. J Pharmacol Exp Ther 1996; 276: 658-666

${ }^{30}$ Kapur S, Zirpusky RB, Remington G. Clinical and theoretical implications of 5-HT2 and D2 receptor occupancy of clozapine, risperidone and olanzapine in schizophrenia. Am J Psychiatry 1998; 155: 921 - 928 ${ }^{31}$ Crawford AM, Beasley CM, Tollefson GD. The acute and long-term effect of olanzapine compared with placebo and haloperidol on serum prolactin concentrations. Schizophr Res 1997; 26: $41-54$

32 Jones B, Basson BR, Walker DJ, Crawford AM, Kinon BJ. Weight change and atypical antipsychotic treatment in patients with schizophrenia. J Clin Psychiatry 2001; 62 (Suppl 2): $41-44$

${ }^{33}$ Kraus T, Zimmermann U, Schuld A, Haack M, Hinze-Selch D, Pollmächer T. Zur Pathophysiologie der Gewichtsregulation im Rahmen der Therapie mit Psychopharmaka. Fortschr Neurol Psychiatr 2001; 69: 116 - 137

${ }^{34}$ Kraus T, Haack M, Schuld A, Hinze-Selch D, Kuhn M, Uhr M, Pollmächer T. Body weight and leptin plasma levels during treatment with antipsychotic drugs. Am J Psychiatry 1999; 156: 312 - 314

${ }^{35}$ Hinze-Selch D, Schuld A, Kraus T, Kuhn M, Uhr M, Haack M, Pollmächer T. Effects of antidepressants on weight and on the plasma levels of leptin, TNF-alpha and soluble TNF receptors: A longitudinal study in patients treated with amitriptyline or paroxetine. Neuropsychopharmacology 2000; 23: 13-19

${ }^{36}$ Allison DB, Mentore JL, Heo M, Chandler LP, Cappelleri JC, Infante MC, Weiden PJ. Antipsychotic-induced weight gain: a comprehensive research synthesis. Am J Psychiatry 1999; 156: 1686 - 1696

37 Agelink MW, Majewski T, Wurthmann C, Lukas K, Ullrich H, Linka T, Klieser E. Effects of newer atypical antipsychotics on autonomic neurocardiac function: a comparison between amisulpride, olanzapine, sertindole, and clozapine. J Clin Psychopharmacol 2001; 21: 8-13

${ }^{38}$ Czekalla J, Kollack-Walker S, Beasley jr CM. Cardiac safety parameters of olanzapine: comparison with other atypical and typical antipsychotics. J Clin Psychiatry 2001; 62 (Suppl 2): 35-40

${ }^{39}$ Robertson MD, McMullin MM. Olanzapine concentrations in clinical serum and postmortem blood specimens - when does therapeutic become toxic? J Forensic Sci 2000; 45: 418-421

${ }^{40}$ Wong SHY, Gock SB, Venuti SE, Stormo KA, Greenbaum J, Biedrzycki L, Jentzen JM. Olanzapine associated deaths: a report of five cases. Proc SOFT-TIAFT Meeting, 1998

${ }^{41}$ Levine BS, Wu SC, Smialek JE. Olanzapine concentrations in forensic investigations. Proc SOFT-TIAFT Meeting 1998: 245-249

${ }^{42}$ Anderson DT, Kuwahara T. Thirty-five case studies involving postmortem tissue distribution of olanzapine (Zyprexa). Proc SOFT-TIAFT Meeting 1998: $236-244$

43 Arzneimittelkommission der deutschen Ärzteschaft. Blutbildveränderungen unter dem neueren atypischen Neuroleptikum Olanzapin. Dtsch Aerztbl 2001; 3: 130

${ }^{44}$ Naumann R, Felber W, Heilemann H, Reuster T. Olanzapine-induced agranulocytosis. Lancet 1999; 354: 566-567

${ }^{45}$ Benedetti F, Cayallaro R, Smeraldi E. Olanzapine-induced neutropenia after clozapine-induced neutropenia. Lancet 1999; 354: 567

${ }^{46}$ Steinwachs A, Grohmann R, Pedrosa F, Rüther E, Schwerdtner I. Two cases of olanzapine-induced reversible neutropenia. Pharmacopsychiatry 1999; 32: 154-156

${ }^{47}$ Dettling M, Cascorbi I, Hellweg R, Deicke U, Weise L, Müller-Oerlinghausen $\mathrm{B}$. Genetic determinants of drug induced agranulocytosis: potential risk of olanzapine? Pharmacopsychiatry 1999; 32: 110 - 112

48 Schuld A, Kraus T, Hinze-Selch D, Haack M, Pollmächer T. Granulocyte colony-stimulating factor plasma levels during clozapine- and olanzapine-induced granulocytopenia. Acta Psychiatr Scand 2000; 102 : $153-155$

${ }^{49}$ Perry PJ, Sanger T, Beasley C. Olanzapine plasma concentrations and clinical response in acutely ill schizophrenic patients. J Clin Psychopharmacol 1997; 17: $472-477$

50 Perry PJ, Lund BC, Sanger T, Beasley C. Olanzapine plasma concentrations and clinical response: acute phase results of the North American olanzapine trial. J Clin Psychopharmacol 2001; 21: 14-20 